\title{
Concentration Compactness at the Mountain Pass Level in Semilinear Elliptic Problems
}

\author{
Kyril Tintarev
}

\begin{abstract}
Let $c$ be the usual mountain pass level for the semilinear elliptic functional

$$
G(u)=\frac{1}{2} \int_{\Omega}\left(|\nabla u|^{2}+\lambda u^{2}\right) d x-\int_{\Omega} F(x, u(x)) d x .
$$

In general, $c \leq c_{\#}$, where $c_{\#}$ is the analogous mountain pass level of the asymptotic functional $G_{\#}$ defined with respect to unbounded shifts or dilations. We show under general conditions that whenever the strict inequality $c \leq c_{\#}$ holds, the functional $G$ satisfies the Palais-Smale condition at the level $c$ and, consequently, has a critical point at this level. This sets a solvability framework for unconstrained mountain pass similar to that of P.-L.Lions set for constrained minimization. The nonlinearity $F$ is allowed to have critical growth with asymptotically selfsimilar oscillations about the critical "stem" $|u|^{2^{*}}$ and not only "stem" asymptotics. For example, the main existence result, Theorem 3.2 , holds for $F(x, s)=|s|^{2^{*}} e^{\frac{\sigma_{N} x^{2}}{1+x^{2}} \sin (2 \log (|s|))}$. Since the unconstrained minimax is studied, the convexity-type conditions that arise with the use of Nehari constraint $\left(G^{\prime}(u), u\right)=0$ are not required.
\end{abstract}

Mathematics Subject Classification (2000). Primary 35J20, 35J60 Secondary 49J35.

Keywords. Semilinear elliptic equations, concentration compactness, mountain pass, positive solutions, variational problems.

\section{Introduction}

In this paper we study existence for the classical semilinear elliptic problem in a domain $\Omega \subset \mathbb{R}^{N}$,

$$
-\Delta u+\lambda u=f(x, u),
$$

Research done as a visitor at Ceremath, University of Toulouse 1. 
with the Dirichlet boundary condition. The number $\lambda$ is assumed to be at or above the bottom of the spectrum for the Dirichlet Laplacian in $\Omega$. We consider here cases where the correspondent Sobolev imbedding lacks compactness, namely when $\Omega=\mathbb{R}^{N}$ or when the nonlinearity $f(x, s)$ has the growth of the critical magnitude $|s|^{2^{*}-1}$, where $2^{*}=\frac{2 N}{N-2}$ and $N>2$. The variational framework set by Ambrosetti and Rabinowitz in [1], initially for the case of bounded $\Omega$ and the subcritical nonlinearity, where one can benefit from compactness of Sobolev imbeddings, faces significant difficulties when the the problem lacks compactness, requiring the concentration compactness argument. The literature on this topics is extensive and contains a variety of solvability conditions that is often hard to compare. Below is a partial survey of known results. The purpose of this paper is to provide more general existence results under weak and transparent assumptions, based on the standard, unconstrained, mountain pass setting.

\subsection{Problems with homogeneity}

If $f(x, u)=a(x)|u|^{p-2} u$, solutions to (1.1), can be obtained by constrained minimization

$$
c=\inf _{u \neq 0} \frac{\int_{\Omega}\left(|\nabla u|^{2}+\lambda u^{2}\right) d x}{\left(\int_{\Omega} a(x)|u|^{p} d x\right)^{2 / p}} .
$$

Homogeneity of the nonlinearity means that one can replace the Lagrange multiplier for the minimizer with 1 by multiplying the minimizer of (1.2) by an appropriate positive constant. Since the loss of compactness in these problems is due to non-compact transformations (shifts or dilations), discrete sequences of these transformation define asymptotic problems (or problems at infinity). Applying such sequences one can immediately see that the inequality $c \leq c_{\infty}$, where $c_{\infty}$ is the constrained minimum for an asymptotic counterpart of (1.2), is always true. In the founding papers P.L.-Lions on concentration compactness [22-25], the strict inequality $c<c_{\infty}$ was employed as a sufficient existence condition to prove existence minimizers in $(1.2)$ both in the subcritical $\left(p<2^{*}\right)$ and the critical $\left(p=2^{*}\right.$, in particular the celebrated Brezis-Nirenberg case $\lambda<0, a=1, N>2, p=2^{*}$ on bounded domains, [8]).

In the autonomous $(f(x, s)=f(s))$ subcritical case on $\mathbb{R}^{N}$, the imbedding of the subspace of $H^{1}$ - radially symmetric functions into $L^{p}$ is compact [17], so that existence of minimizers in (1.2) follows from a standard weak continuity argument. Lagrange multipliers here can be set to 1 even for general nonlinearities $f(s)$ of subcritical growth, since autonomous problems on $\mathbb{R}^{N}$ possess additional homogeneity, namely the one with respect to the transformation $s \mapsto u(\cdot / s)$. Existence results for the subcritical autonomous case are due to Berestycki and Lions [5]. In the critical case the imbedding of the subspace of radial functions into $L^{2^{*}}$ is no longer compact, and concentration compactness argument was used by Lions [24] and Flucher and Müller [19] to proved existence of minimizers for general autonomous nonlinearity $f$ under the penalty condition $c<c_{\infty}$, which is realized, 
in terms of the Lagrangian density

$$
F(t) \stackrel{\text { def }}{=} \int_{0}^{t} f(s) d s
$$

as $F(t)>F_{\infty}(t)$, where $F_{\infty}$ is the appropriate asymptotic counterpart of $F$.

In the case of subcritical nonlinearity, more refined realizations of the penalty condition $c<c_{\infty}$ are given by Sirakov [30]. The best known case when the condition $F>F_{\infty}$ does not yield $c<c_{\infty}$ is the Brezis-Nirenberg problem on bounded domains with $N=3$. The reason to it is that the relevant asymptotic problem is a problem in $\mathbb{R}^{N}$ rather than in the original domain and multiplication of the solution of the asymptotic problem with a cut-off function when $N>3$ yields an irredeemable error: indeed, [8] provides a non-existence counterexample.

\subsection{Problems without homogeneity}

In problems without homogeneity there is generally no obvious way to attain a specified value of Lagrange multiplier, even by varying the level of the constraint. This approach may be still practical when the minimization of the functional

$$
G(u)=\frac{1}{2} \int_{\Omega}\left(|\nabla u|^{2}+\lambda u^{2}\right) d x-\int_{\Omega} F(x, u(x)) d x,
$$

whose Euler-Lagrange equation is (1.1) is done under the removable (meaning a zero Lagrange multiplier) Nehari constraint $\left(G^{\prime}(u), u\right)=0$. A typical sufficient condition that allows to use the Nehari constraint is monotonicity of $s \mapsto f(x, s) / s$. We refer the reader to typical results on this lines in Chapter 4 in the book of Willem [35]). In this paper we opt to study the unconstrained mountain pass.

Below we use the following terminology. A number $c \in \mathbb{R}$ is called a critical value of a $C^{1}$ - functional $G$ in a Banach space, if it is the value $G(u)$ at a critical point $u$ (that is, when $G^{\prime}(u)=0$ ), and it is called a critical level of a functional if there is a sequence $u_{k}$, called critical sequence, such that $G\left(u_{k}\right) \rightarrow c$ and $G^{\prime}\left(u_{k}\right) \rightarrow$ 0 . The Palais-Smale condition $(P S)_{c}$ says that every critical sequence at the level $c$ has a convergent subsequence, in which case the critical level becomes a critical value.

There is a general result on weak convergence of critical sequences for semilinear elliptic equations to a non-zero solution due to Rabinowitz [28], but his proof does not verify $(P S)_{c}$ condition.

There are several important reasons to verify $(P S)_{c}$, such as: utility of minimax statements to calculate or estimate critical values; sorting obtained by different methods by the values of the functional; estimating the Morse index of a critical point; and existence of multiple critical points via minimax principles that require the $(P S)_{c}$. On the other hand, the $(P S)_{c}$-condition in non-compact problems is well known to fail on critical levels produced by divergent bounded sequences of 
the form

$$
u_{k}=w+\sum_{n=1}^{m} g_{k}^{(n)} w_{\infty},
$$

where $g_{k}^{(n)}$ are pairwise asymptotically orthogonal sequences of transformations responsible for the loss of compactness (actions of translations or dilations), $w$ is a critical point of the functional and $w_{\infty}$ is a critical point of the asymptotic problem.

Our approach is based on the observation that (1.4) is essentially the only way a bounded critical sequence can diverge, which leads to a conclusion that $(P S)_{c}$ in the Ambrosetti-Rabinowitz settings holds whenever the mountain pass level satisfies $c<c_{\infty}$ (while $c \leq c_{\infty}$ is true in general). Decompositions of critical sequences similar to (1.4) have been introduced by Struwe [31] for the critical exponent case in bounded domains, followed by Brezis and Coron [7] and by Lions [26] for subcritical problems in $\mathbb{R}^{N}$ and numerous authors afterward. We use the version of the "multibump" decomposition, Theorem 6.1 [34, Theorem 5.1] that is not restricted to critical sequences of a specific functional. In restriction to critical sequences this is close, up to elementary modifications, to the "splitting lemma" in Benci and Cerami [4].

Verification of Palais-Smale condition at the mountain pass level is trivial when the problem has homogeneity and thus has an equivalent constrained minimization statement. This has been already observed by Cerami, Fortunato and Struwe [11] who considered autonomous problem with the critical stem nonlinearity on bounded domains in the mountain pass setting, noting that the solvability condition $c<c_{\infty}$, established by Lions, extends to the mountain pass problems. Chabrowski and Yang [14] have verified the Palais-Smale condition in the subcritical case under an additional assumption $f_{\infty}(s) / s$ increasing for an interval of critical values $\left(0, J^{\infty}\right)$, where $J^{\infty}$ is a constrained minimum of $G_{\infty}$ under the Nehari constraint $\left(G_{\infty}^{\prime}(u), u\right)=0$. Lemma 2.2 in [16] states that $c_{\infty}$ is attained on the straight line path $t \mapsto t w$, where $w$ is, in terms of Chabrowski and Yang, a minimizer for $G_{\infty}$ under the $\mathrm{N}$ ehari constraint, which implies that $J^{\infty}=c_{\infty}$. In other words, the solvability condition of Chabrowski and Yang is an implicit form of the condition $c<c_{\infty}$, used in this paper. We extend (in Section 5) their result to the case when monotonicity of $f_{\infty}(s) / s$, assumed by Chabrowski and Yang, is no longer required. Another related result for the subcritical case is due to Bartsch and Wang [3], but it is out of scope of this paper, as it deals with the equation $-\Delta u+V(x) u=f(x, u)$ for general subcritical $f$, deriving the Palais-Smale condition from unbounded (not necessarily coercive) $V>0$, namely such that for every $M>0$, the set $V^{-1}(0, M)$ has a finite measure. We do not survey the literature here for the critical case, referring the reader to the bibliography in the books of Chabrowski, [10], Flucher [20] and Willem [35] and to the recent survey of Bartsch, Wang and Willem [2]. As a rule, the nonlinearity considered in literature is of the form $a(x)|u|^{2^{*}}$ plus a subcritical term, and this paper considers a more general case. 
Our paper is organized as follows. For the sake of simplicity, we assume that the nonlinearity $F(x, s)$ is continuously differentiable with respect to $s$ and that its derivative admits required asymptotic functions as uniform limits, so the asymptotic functionals and the asymptotic equations are well defined. Section 2 gives a generalization of the existence result of Lions and Flucher \& Müller in the sense that the nonlinearity at infinity is defined due to discrete dilations, which generally gives a smaller $F_{\infty}$ (involved in the penalty condition $F>F_{\infty}$ ) than the upper limit defined in [19]. In particular, we consider a nonlinearity $F$ such that $t \mapsto F\left(e^{t}\right) e^{-2^{*} t}$ is a periodic function, that is, a nonlinearity with regular oscillations about the critical "stem" $s^{2^{*}}$. Existence of solutions in the zero mass case $(\lambda=0)$ requires some oscillations of this type with necessity due to Pohožaev identity $\left([27]\right.$ for bounded domain and $[5]$ for $\mathbb{R}^{N}$ )

$$
\int_{\mathbb{R}^{N}}|\nabla u|^{2} d x=2^{*} \int_{\mathbb{R}^{N}}\left(F(u)-\lambda u^{2}\right) d x .
$$

(Validity of this identity requires that $u$ and its gradient are decaying sufficiently fast at infinity. These decay rates are verified in the case $\lambda>0$ with subcritical nonlinearity in [5] and for $\lambda=0$ and the nonlinearity $F$ bounded by the critical stem $C|u|^{2^{*}}$ in [18].) For positive solutions, Pohožaev identity is equivalent to

$$
\int_{0}^{\infty} s^{2^{*}+1} \frac{d}{d s} \frac{F(s)-\lambda s^{2}}{s^{2^{*}}} d|\{u \leq s\}|=0,
$$

which implies that $\frac{F(s)-\lambda s^{2}}{s^{2 *}}$ is necessarily non-monotone, unless it is a constant. If $\lambda>0$, this relation is satisfied whenever $F(s)=o\left(s^{2}\right)$ at zero and $F(s) / s^{2} \rightarrow \infty$ at infinity, which are the typical sufficient conditions for continuity of $G$ to have the mountain pass geometry. For $\lambda=0$, this condition becomes, however, a significant condition of oscillatory behavior of $F(s) / s^{2^{*}}$.

Critical points obtained in Section 2 are used in calculations of Section 3 dealing with the non-autonomous critical problem in $\mathbb{R}^{N}$ in the zero mass $(\lambda=0)$ case. In Section 4 we deal with critical problems on bounded domains, that is, with a generalization of the Brezis-Nirenberg problem to oscillatory critical nonlinearities. In Section 5 we give an improved existence condition in the subcritical case on $\mathbb{R}^{N}$. The main results of this paper are existence statements Theorem 3.2, Theorem 4.2 and Theorem 5.2. A typical example of nonlinearity for which we establish existence of a critical point at the unconstrained mountain pass level is

$$
F(x, s)=F_{1}(x, s)+|s|^{2^{*}} e^{\frac{\sigma_{N} x^{2}}{1+x^{2}} \sin (2 \log (|s|))},
$$

for $N>2, \sigma_{N}>0$ sufficiently small and $F_{1}$ is a general subcritical nonlinearity satisfying conditions of Theorem 3.2, for example

$$
F_{1}(x, s)=g(x)|s|^{p}
$$

where $p \in\left(2,2^{*}\right)$ and $g(x) \in C\left(\mathbb{R}^{N}\right)$ satisfies $0<\lim _{|x| \rightarrow \infty} g(x)<g(y), y \in \mathbb{R}^{N}$.

For the sake of consistency we also include for each of these three cases an elementary statement that the non-strict version $c \leq c_{\infty}$ of the penalty condition is unconditionally true. 


\section{Preliminaries: Autonomous critical problem}

We consider the space $\mathcal{D}^{1,2}\left(\mathbb{R}^{N}\right), N>2$, a completion of $C_{0}^{\infty}(\Omega)$ with respect to the gradient norm

$$
\|u\|=\left(\int_{\mathbb{R}^{N}}|\nabla u|^{2} d x\right)^{1 / 2},
$$

and we equip the space $\mathcal{D}^{1,2}\left(\mathbb{R}^{N}\right)$ with the group $D(N, \mathbb{Z}, \gamma)$ of unitary operators generated by the shifts

$$
D_{\mathbb{R}^{N}} \stackrel{\text { def }}{=}\left\{u \mapsto u(\cdot-y), y \in \mathbb{R}^{N}\right\}
$$

and by the action of discrete dilations with a fixed scaling factor $\gamma>1$,

$$
\delta_{\mathbb{Z}, \gamma} \stackrel{\text { def }}{=}\left\{u \mapsto \gamma^{\frac{N-2}{2} j} u\left(\gamma^{j} \cdot\right), j \in \mathbb{Z}\right\} .
$$

Let $f \in C(\mathbb{R})$ and let

$$
F(s) \stackrel{\text { def }}{=} \int_{0}^{s} f(\sigma) d \sigma
$$

Assuming

$$
|F(s)| \leq C|s|^{2^{*}}
$$

we set

$$
\psi(u) \stackrel{\text { def }}{=} \int_{\mathbb{R}^{N}} F(u) d x
$$

and

$$
G(u) \stackrel{\text { def }}{=} \frac{1}{2}\left\|u^{2}\right\|-\psi(u),
$$

and note that $G \in C\left(\mathcal{D}^{1,2}\left(\mathbb{R}^{N}\right)\right)$. Let

$$
\Phi_{G}=\left\{\varphi \in C\left([0, \infty) \rightarrow H^{1}\left(\mathbb{R}^{N}\right)\right): \varphi_{0}=0, \lim _{t \rightarrow \infty} G\left(\varphi_{t}\right)=-\infty\right\} .
$$

If $\Phi_{G}=\emptyset$, we set $c(G)=+\infty$, otherwise

$$
c(G) \stackrel{\text { def }}{=} \inf _{\varphi \in \Phi_{G}} \sup _{t \in[0, \infty)} G\left(\varphi_{t}\right) .
$$

Proposition 2.1. The set $\Phi_{G}$ is nonempty if and only if $\sup F>0$, in which case $\Phi_{G}$ contains a path $u_{t}(x)=u(x / t)$ with $u \in C_{0}^{\infty}\left(\mathbb{R}^{N}\right)$ and $\psi(u)>0$.

Proof. Note first that the path $u_{s}$ is a continuous map from $(0, \infty)$ to $\mathcal{D}^{1,2}\left(\mathbb{R}^{N}\right)$ that extends by continuity as $u_{0}=0$ : $\left\|u_{s}\right\|^{2}=s^{N-2}\|u\|^{2}$, and in particular $\lim _{s \rightarrow 0}\left\|u_{s}\right\|=0$. Since the norm is continuous, it suffices to prove continuity of $u_{s}$ in $\mathcal{D}^{\prime}\left(\mathbb{R}^{N}\right)$. Indeed, with $\varphi \in C_{0}^{\infty}(\Omega)$ and $s, s_{0}>0$, by Lebesgue convergence theorem,

$$
\int u_{s} \varphi=s^{N} \int u \varphi(s \cdot) \rightarrow s_{0}^{N} \int u \varphi\left(s_{0} \cdot\right) \quad \text { as } \quad s \rightarrow s_{0} \cdot
$$


If $\sup F>0$, then $\sup \psi>0$ and $\Phi_{G} \neq \emptyset$, since it contains a path $u_{s}(x)=$ $u(x / s)$ with $\psi(u)>0$. Indeed, by the change of variables in respective integrals,

$$
G\left(u_{s}\right)=\frac{1}{2} s^{N-2}\|u\|^{2}-s^{N} \psi(u) .
$$

If, on the other hand, $F \leq 0$, then $G \geq 0$ and $\Psi_{G}=\emptyset$.

We assume that the following limits exist:

$$
\begin{aligned}
& f_{+}(s) \stackrel{\text { def }}{=} \lim _{j \in \mathbb{Z}, j \rightarrow+\infty} \gamma^{\frac{N+2}{2} j} f\left(\gamma^{-\frac{N-2}{2} j} s\right), \\
& f_{-}(s) \stackrel{\text { def }}{=} \lim _{j \in \mathbb{Z}, j \rightarrow-\infty} \gamma^{\frac{N+2}{2} j} f\left(\gamma^{-\frac{N-2}{2} j} s\right) .
\end{aligned}
$$

Repeating the definitions above for the functions $f_{ \pm}$, we consider $F_{ \pm}, \psi_{ \pm} G_{ \pm}, \Phi_{G_{ \pm}}$ and $c\left(G_{ \pm}\right)$. We will call the function $F$ selfsimilar with a factor $\gamma$ if

$$
F(s)=\gamma^{-N j} F\left(\gamma^{\frac{N-2}{2} j} s\right), \quad j \in \mathbb{Z}, \quad s \in \mathbb{R} .
$$

It is uniquely defined by its values on the intervals $(1, \gamma)$ and $(-\gamma,-1)$. If $F$ is differentiable, one obviously has

$$
f(s)=\gamma^{-\frac{N+2}{2} j} f\left(\gamma^{\frac{N-2}{2} j} s\right), \quad j \in \mathbb{Z}, \quad s \in \mathbb{R} .
$$

Note that for any given $F$ that admits asymptotic functions $F_{ \pm}$, they are selfsimilar.

The following statement generalizes Theorem 5.2 from [34].

Proposition 2.2. Assume (2.1) and assume, for each of the signs "+" and "-", that either $\sup F_{ \pm}>0$, or $F_{ \pm}=0$ with $\sup F>0$. Let

$$
\kappa_{t} \stackrel{\text { def }}{=} \sup _{\|u\|^{2}=t} \psi(u), \quad t>0
$$

and let $\kappa_{t, \pm}$ be the value (2.8) corresponding to the functionals $\psi_{ \pm}$. If $F$ satisfies (2.6) with some $\gamma>1$ or $\kappa>\max \left\{\kappa_{1,-}, \kappa_{1,+}\right\}$, then the maximum in (2.8) is attained.

Furthermore, the inequality $\kappa_{t}>\kappa_{t, \pm}$ holds whenever $F \geq F_{ \pm}$with the strict inequality in a neighborhood of zero.

Proof. 1. By substitution $u(s)=v\left(s / t^{\frac{1}{N-2}}\right)$

$$
\kappa_{t}=\kappa_{1} t^{\frac{2^{*}}{2}},
$$

so it suffices to prove the lemma for $t=1$.

2. Assume now that $F$ satisfies (2.6). If $F=0$, then $\kappa_{1}=0$ and any function with the given norm is a maximizer for $\psi$. Assume now that $\sup F>0$. Let $u_{k}$ be a maximizing sequence in (2.8), that is, $\left\|u_{k}\right\|^{2}=1$ and $\psi\left(u_{k}\right) \rightarrow \kappa_{1}$. Let 
$y_{k}^{(n)} \in \mathbb{R}^{N}, j_{k}^{(n)} \in \mathbb{Z}, w^{(n)} \in \mathcal{D}^{1,2}\left(\mathbb{R}^{N}\right)$ and the index sets $\mathbb{N}_{+\infty}, \mathbb{N}_{-\infty}, \mathbb{N}_{0} \subset \mathbb{N}$ be as in Theorem 6.1. Note that by Lemma 6.2,

$$
\kappa_{t}=\lim \psi\left(u_{k}\right)=\sum_{n \in \mathbb{N}} \psi\left(w^{(n)}\right) .
$$

At the same time from (6.3)

$$
1=\left\|u_{k}\right\|^{2} \geq \sum_{n \in \mathbb{N}}\left\|w^{(n)}\right\|^{2}
$$

L et $v^{(n)}(x)=w^{(n)}\left(s_{n} x\right)$ with $s_{n}=\left\|w^{(n)}\right\|^{\frac{2}{N-2}}$. Then $\left\|v^{(n)}\right\|^{2}=1$ whenever $w^{(n)} \neq 0$, otherwise $v^{(n)}=0$. Rewriting relation (2.10) in terms of $v^{(n)}$ gives

$$
\kappa_{1}=\sum_{n \in \mathbb{N}} s_{n}^{N} \psi\left(v^{(n)}\right) \leq \kappa_{1} \sum_{n \in \mathbb{N}} s_{n}^{N},
$$

or, in other words,

$$
\sum_{n \in \mathbb{N}} s_{n}^{N} \geq 1
$$

On the other hand, from (2.11) we derive

$$
\sum_{n \in \mathbb{N}} s_{n}^{N-2} \leq 1
$$

Relations (2.13) and (2.14) can hold simultaneously if and only if there is a $n_{0} \in \mathbb{N}$ such that $s_{n_{0}}=1$, while $s_{n}=0$ whenever $n \neq n_{0}$. Consequently, we have from (6.4) $u_{k}-\gamma^{\frac{N-2}{2} j_{k}^{\left(n_{0}\right)}} w^{\left(n_{0}\right)}\left(\gamma^{j_{k}^{\left(n_{0}\right)}}\left(\cdot-y_{k}^{\left(n_{0}\right)}\right)\right) \rightarrow 0$ in $L^{2^{*}}\left(\mathbb{R}^{N}\right)$, or, by replacing the maximizing sequence $u_{k}$ with the maximizing sequence $\hat{u}_{k} \stackrel{\text { def }}{=}$ $\gamma^{-\frac{N-2}{2} j_{k}^{\left(n_{0}\right)}} u_{k}\left(\gamma^{-j_{k}^{\left(n_{0}\right)}} \cdot+y_{k}^{\left(n_{0}\right)}\right)$, we have $\hat{u}_{k} \rightarrow w^{\left(n_{0}\right)}$ in $L^{2^{*}}\left(\mathbb{R}^{N}\right)$. At the same time, by the weak lower semicontinuity of norms, $\left\|w^{\left(n_{0}\right)}\right\|^{2} \leq 1$, while by continuity of $\psi$ in $L^{2^{*}}\left(\mathbb{R}^{N}\right)$ we have $\psi\left(w^{\left(n_{0}\right)}\right)=\kappa_{1}$. Since the latter can hold only when $\left\|w^{\left(n_{0}\right)}\right\|^{2}=1, w^{\left(n_{0}\right)}$ is the desired maximizer.

3. Consider now the general case with $\kappa_{1}>\kappa_{1, \pm}$ and note that, since $F_{ \pm}$ satisfies (2.6), the maximum for $\kappa_{1, \pm}$ is attained due to the previous step. Let $u_{k}$ be a minimizing sequence in $(2.8)$ and let $y_{k}^{(n)} \in \mathbb{R}^{N}, j_{k}^{(n)} \in \mathbb{Z}, w^{(n)} \in \mathcal{D}^{1,2}\left(\mathbb{R}^{N}\right)$ and let the index sets $\mathbb{N}_{+\infty}, \mathbb{N}_{-\infty}, \mathbb{N}_{0} \subset \mathbb{N}$ be as in Theorem 6.1. Note that by Lemma 6.2,

$$
\kappa_{t}=\lim \psi\left(u_{k}\right)=\sum_{n \in \mathbb{N}_{0}} \psi\left(w^{(n)}\right)+\sum_{n \in \mathbb{N}_{-}} \psi_{-}\left(w^{(n)}\right)+\sum_{n \in \mathbb{N}_{+\infty}} \psi_{+}\left(w^{(n)}\right) .
$$

Let, as in the step $2, v^{(n)}(x)=w^{(n)}\left(s_{n} x\right)$ with $s_{n}=\left\|w^{(n)}\right\|^{\frac{2}{N-2}}$, which yields

$$
\sum_{n \in \mathbb{N}_{0}} s_{n}^{N}+\frac{\kappa_{1,-}}{\kappa_{1}} \sum_{n \in \mathbb{N}_{-\infty}} s_{n}^{N}+\frac{\kappa_{1,+}}{\kappa_{1}} \sum_{n \in \mathbb{N}_{+\infty}} s_{n}^{N} \geq 1,
$$

while we still have (2.14). Since $\frac{\kappa_{1, \pm}}{\kappa_{1}}<1$, relations (2.16) and (2.14) can hold simultaneously if and only if there is a $n_{0} \in \mathbb{N}_{0}$ such that $s_{n_{0}}=1$, while $s_{n}=0$ 
whenever $n \neq n_{0}$. Consequently, we have from (6.4) $u_{k}-w^{\left(n_{0}\right)}\left(\cdot-y_{k}^{\left(n_{0}\right)}\right) \rightarrow 0$ in $L^{2^{*}}\left(\mathbb{R}^{N}\right)$. Similarly to the step 2 we conclude that $w^{\left(n_{0}\right)}$ is the desired maximizer.

4. The last assertion of the proposition is obvious if we take into account that the range of any function in $\mathcal{D}^{1,2}\left(\mathbb{R}^{N}\right)$ is a connected set whose closure contains zero.

Remark 2.3. Note that one always has $\kappa_{t} \geq \kappa_{t, \pm}$, since if $w$ is a maximizer for $\kappa_{1, \pm}$, then $\psi\left(\gamma \frac{N-2}{2} j w\left(\gamma^{j} x\right)\right) \rightarrow \psi_{ \pm}(w)$ as $j \in \mathbb{Z}, j \rightarrow \pm \infty$.

If $w_{1}$ is a maximizer of (2.8) for $t=1$, then, obviously, the function

$$
w_{t}(x)=w_{1}\left(t^{-\frac{1}{N-2}} x\right)
$$

is a maximizer for $t>0$.

We now connect the maximizers (2.8) with the mountain pass values for $G$.

Proposition 2.4. Assume that $\sup F>0$ and that

$$
|f(s)| \leq C|s|^{2^{*}-1} \text {. }
$$

Then any maximizer $w$ for (2.8) corresponding to

$$
t=t_{0} \stackrel{\text { def }}{=}\left(2^{*} \kappa_{1}\right)^{-\frac{2}{N-2}},
$$

is a critical point of $G$. Moreover,

(i) $0<G(w)=c(G)=\max _{t>0} G(w(\cdot / t))$; and

(ii) if $v$ is a critical point of $G$ such that $\psi(v)>0$, then $G(v) \geq c(G)$.

Proof. From (2.17) follows that $G \in C^{1}\left(\mathcal{D}^{1,2}\left(\mathbb{R}^{N}\right)\right)$. We prove (ii) first. Let $v$ be any critical point with $\psi(v)>0$ and let $v_{s}(x)=v(x / s)$. Since $\psi(v)>0$, we have $v_{s} \in \Phi_{G}$ and the function $s \mapsto G\left(v_{s}\right)=\frac{1}{2} s^{N-2}\|v\|^{2}-s^{N} \psi(v)$ has a single critical point, a maximum, which is necessarily attained at $s=1$ since $G^{\prime}(v)=0$. Then

$$
c(G) \leq \max _{s \geq 0} G\left(v_{s}\right)=G(v),
$$

which verifies (ii).

Assume now that (2.8) has a maximizer $w$. By Remark 2.3, $w$ is a critical point of $G$. By the argument above, $c(G) \leq G(w)$. On the other hand, since any path $u_{s} \in \Phi_{G}$ starts at the origin and is unbounded, we have, using the notation $\hat{u}=u\left(\|u\|^{-\frac{2}{N-2}} \cdot\right), r_{s}=\left\|u_{s}\right\|$, and taking into account that $\|\hat{u}\|=1$,

$$
\begin{aligned}
c(G) & =\inf _{u_{s} \in \Phi} \max _{s \geq 0} \frac{1}{2} r_{s}^{2}-r_{s}^{\frac{2 N}{N-2}} \psi\left(\hat{u}_{s}\right) \\
& \geq \inf _{u_{s} \in \Phi} \max _{s \geq 0} \frac{1}{2} r_{s}^{2}-r_{s}^{\frac{2 N}{N-2}} \kappa_{1} \\
& =\max _{r \geq 0} \frac{1}{2} r^{2}-r^{\frac{2 N}{N-2}} \kappa_{1}=G(w) .
\end{aligned}
$$

We conclude that $c(G)=G(w)$ and the path $w_{t} \in \Phi_{G}$ is a minimal path passing through the critical point $w$. Explicit calculation of the maximum on the minimal path gives the value of $t_{0}$. This proves (i). 


\section{Non-autonomous critical problem in $\mathbb{R}^{N}$}

Let now $f(x, s) \in C\left(\mathbb{R}^{N} \times \mathbb{R}\right), N>2$.

Assume that for some $\gamma>1$ the following limits exist and that the convergence is uniform:

$$
\begin{aligned}
& f_{0}(s) \stackrel{\text { def }}{=} \lim _{|x| \rightarrow \infty} f(x, s), \\
& f_{-}(s) \stackrel{\text { def }}{=} \lim _{j \in \mathbb{Z}, j \rightarrow-\infty} \gamma^{\frac{N+2}{2} j} f\left(\gamma^{j} x, \gamma^{-\frac{N-2}{2} j} s\right), \\
& f_{+}(s) \stackrel{\text { def }}{=} \lim _{j \in \mathbb{Z}, j \rightarrow+\infty} \gamma^{\frac{N+2}{2} j} f\left(\gamma^{j} x, \gamma^{-\frac{N-2}{2} j} s\right) .
\end{aligned}
$$

Let

and assume the inequality

$$
F(x, s)=\int_{0}^{s} f(x, \sigma) d \sigma,
$$

$$
|f(x, s)| \leq C|s|^{2^{*}-1}, \quad s \in \mathbb{R}, \quad x \in \mathbb{R}^{N} .
$$

Let

$$
\begin{aligned}
\psi(u) & =\int_{\mathbb{R}^{N}} F(x, u) d x, \\
G(u) & =\frac{1}{2}\|u\|_{\mathcal{D}^{1,2}\left(\mathbb{R}^{N}\right)}^{2}-\psi(u),
\end{aligned}
$$

and let $\Phi_{G}$, and $c(G)$ be as in (2.4) and in (2.5) respectively. We will also consider similarly defined respective $F_{\#}, \psi_{\#}, G_{\#}, \Phi_{G_{\#}} \Phi_{G_{\#}}$ and $c\left(G_{\#}\right)$, where \# will refer in what follows to any of the three indices $0,+,-$.

Proposition 3.1. Assume (3.1). Then

$$
c(G) \leq c\left(G_{\#}\right) .
$$

Proof. If $F_{\#} \leq 0$, then $\Phi_{G_{\#}}=\emptyset, c\left(G_{\#}\right)=+\infty$ and the statement is tautological. We may assume now that $\sup F_{\#}>0$. By Proposition 2.4, each of the functionals $G_{ \pm}$has a critical point $w_{ \pm}$on a minimal path $w_{ \pm}(\cdot / s)$. If the functional $G_{0}$ does not have a critical point, from Proposition 2.2 and Proposition 2.4 it follows that its associated constrained supremum $\kappa_{t}$ equals to one of its asymptotic functionals $\psi_{ \pm}$, which are in fact are given by the original $F_{ \pm}$and consequently, we have $c\left(G_{0}\right)=$ $c\left(G_{+}\right)$or $c\left(G_{0}\right)=c\left(G_{-}\right)$. In this case the inequality $c(G)<c\left(G_{0}\right)$ tautologically follows from the inequalities with $G_{\#}=G_{ \pm}$for which critical points do exist. Thus, without loss of generality we may assume that $G_{\#}$ has a critical point $w$ with a minimal path $w(\cdot / s) \in \Phi_{G_{\#}}$. Then there is a sequence of $j_{k} \in \mathbb{Z}$ and $y_{k} \in \mathbb{R}^{N}$ such that either $j_{k} \rightarrow+\infty$, or $j_{k} \rightarrow-\infty$, or $\left|y_{k}\right| \rightarrow \infty$, and such that, with

$$
w_{t ; k} \stackrel{\text { def }}{=} \gamma^{\frac{N-2}{2} j_{k}} w_{t}\left(\gamma^{j_{k}} \cdot+y_{k}\right),
$$

the sequence $G\left(w_{t ; k}\right)$ converges to $G_{\#}\left(w_{t}\right)$ uniformly in $t$. Indeed, with

$$
F_{k}(x, s)=\gamma^{N j_{k}} F\left(\gamma^{-j_{k}}\left(x-y_{k}\right), \gamma^{\frac{N-2}{2} j_{k}} s\right),
$$


it is easy to see that for any $\epsilon>0$ there is a $k_{\epsilon} \in \mathbb{N}$ so that for all $k>k_{\epsilon}$ and $t \geq \epsilon$,

$$
\psi\left(w_{t ; k}\right)=\int_{\mathbb{R}^{N}} F\left(x, w_{t ; k}\right)=t^{N} \int_{\mathbb{R}^{N}} F_{k}(t x, w) \rightarrow t^{N} \psi_{\#}(w)=\psi_{\#}\left(w_{t}\right) .
$$

Let us redefine the path $w_{t ; k}$ for $t \in[0, \epsilon]$, by $\frac{t}{\epsilon} w_{\epsilon ; k}$. Then it is easy to see that with $\epsilon$ sufficiently small,

$$
c(G) \leq \max _{t \geq \epsilon} G\left(w_{t ; k}\right) \rightarrow \max _{t \geq 0} G_{\#}\left(w_{t}\right)=c\left(G_{\#}\right) .
$$

Theorem 3.2. Assume in addition to (3.1) that $F$ is not identically 0 and that

$$
\text { there exists } \mu>2 \text {, such that }
$$

$$
f(x, s) s \geq \mu F(x, s), \quad s \in \mathbb{R}, \quad x \in \mathbb{R}^{N} .
$$

If

$$
c(G)<c\left(G_{\#}\right),
$$

then the functional (3.2) has a critical point at the level $c(G)$ and every critical sequence at this level has a convergent subsequence. Furthermore, the relation (3.4) is satisfied if

$$
F(x, s) \geq F_{\#}(s), \quad x \in \mathbb{R}^{N},
$$

with the strict inequality for $s$ in a neighborhood of zero.

Proof. Since $c(G)<\infty, \sup F>0$, and from the condition $(\mu)$ the mountain pass geometry, that is, $\Psi_{G} \neq \emptyset$ and $c(G)>0$, trivially follows. Note also that one can pass to the limit in $(\mu)$, so the condition holds also for $F_{\#}$. By the standard mountain pass reasoning, the functional $G$ possesses a critical sequence $u_{k} \in \mathcal{D}^{1,2}\left(\mathbb{R}^{N}\right)$, that is, $G\left(u_{k}\right) \rightarrow c(G)$ and $G^{\prime}\left(u_{k}\right) \rightarrow 0$. Also by a standard argument, it follows from $(\mu)$ that the sequence $u_{k}$ is bounded in $\mathcal{D}^{1,2}\left(\mathbb{R}^{N}\right)$. Consider now the renamed subsequence of $u_{k}$ given by Theorem 6.1. If $w^{(n)}=0$ in (6.4) for all $n \geq 2$, then $u_{k} \rightarrow w^{(1)}$ in $L^{2^{*}}, \psi^{\prime}\left(u_{k}\right)$ converges in $\mathcal{D}^{1,2}\left(\mathbb{R}^{N}\right)$, and from $G^{\prime}\left(u_{k}\right) \rightarrow 0$ it follows that $u_{k}$ converges in $\mathcal{D}^{1,2}\left(\mathbb{R}^{N}\right)$ to a critical point of $G$ at the level $c(G)$.

Let us assume now that for some $m \geq 2, w^{(m)} \neq 0$. Due to (6.3) and Lemma 6.5, we have the following estimate of $c(G)$ from below:

$$
\begin{aligned}
c(G) & =\lim G\left(u_{k}\right) \\
& \geq G\left(w^{(1)}\right)+\sum_{n \in \mathbb{N}_{0}} G_{0}\left(w^{(n)}\right)+\sum_{n \in \mathbb{N}_{+\infty}} G_{+}\left(w^{(n)}\right)+\sum_{n \in \mathbb{N}_{-\infty}} G_{-}\left(w^{(n)}\right) .
\end{aligned}
$$

Note that with necessity, $w^{(1)}$ is a critical point of $G$, and $w^{(n)}, n \geq 2$, are critical points of correspondent $G_{\#}$. Let $G_{m}=G_{0}$ if $m \in \mathbb{N}_{0}, G_{m}=G_{+}$if $m \in \mathbb{N}_{+}$and $G_{m}=G_{-}$if $m \in \mathbb{N}_{-}$. The correspondent asymptotic nonlinearity we will denote as $F_{m}$. Due to condition $(\mu), G\left(w^{(1)}\right)=\int\left[\frac{1}{2} f\left(x, w^{(1)}\right) w^{(1)}-F\left(x, w^{(1)}\right)\right] \geq 0$. Similarly $G_{\#}\left(w^{(n)}\right) \geq 0$. Furthermore, since $w^{(m)} \neq 0, \sup F_{m}>0$ and then, for $n=m,(\mu)$ implies $\psi_{m}\left(w^{(m)}\right)>0$ and $G_{m}\left(w^{(m)}\right)>0$. Combining (3.6) with (3.4), we have

$$
G_{\#}\left(w^{(m)}\right)<c\left(G_{\#}\right) .
$$


On the other hand, by Proposition 2.4, $G_{\#}\left(w^{(m)}\right) \geq c\left(G_{\#}\right)$, which is a contradiction. Thus our assumption above that there is such $m \geq 2$ is false. Consequently, $w^{(1)}$ is the desired critical point of $G$.

It remains to verify that (3.5) implies (3.4). If $G_{\#}$ has a critical point $w$ with the critical value $c\left(G_{\#}\right)$ lying on a minimal path $w_{t}(x)=w(x / s)$ (due to Proposition 2.2 this is always the case when \# is a + or a -$)$, then from (3.5) it follows that $\psi(w)>\psi_{\#}(w)$, and then $c(G) \leq \max _{s \geq 0} G\left(w_{s}\right)<\max _{s \geq 0} G_{\#}\left(w_{s}\right)=$ $c\left(G_{\#}\right)$. Assume now that the maximum in (2.8) for $\psi_{\#}$ is not attained, which is, with necessity, the case of $\psi_{0}$. Then, by Proposition 2.2 and Remark 2.3, either $\kappa_{t, 0}=\kappa_{t,+}$ or $\kappa_{t, 0}=\kappa_{t,-}$, which, by Proposition 2.4 gives immediately $c\left(G_{0}\right)=$ $c\left(G_{+}\right)$or $c\left(G_{0}\right)=c\left(G_{-}\right)$. In either case the inequality $c(G)<c\left(G_{0}\right)$ follows from one of the two verified conditions $c(G)<c\left(G_{+}\right)$or $c(G)<c\left(G_{-}\right)$.

\section{Critical case, problems in domains}

Let now $\Omega \subset \mathbb{R}^{N}, N>2$, be a domain with $\bar{\Omega} \neq \mathbb{R}^{N}$. Let $\mathcal{D}_{0}^{1,2}(\Omega)$ be the closure of $C_{0}^{\infty}(\Omega)$ in $\mathcal{D}^{1,2}\left(\mathbb{R}^{N}\right)$. Note that $\mathcal{D}_{0}^{1,2}(\Omega)$ coincides with $\left.H_{0}^{1}(\Omega)\right)$ when $|\Omega|<\infty$, but generally there is no continuous imbedding of $\mathcal{D}_{0}^{1,2}(\Omega)$ into $L^{2}(\Omega)$.

We consider here the functional $(3.2)$, restricted to $\mathcal{D}_{0}^{1,2}(\Omega)$. We assume that $f(x, s) \in C\left(\mathbb{R}^{N} \times \mathbb{R}\right)$ satisfies $(3.1),|\Omega|<\infty$ and

$$
|f(x, s)| \leq C\left(1+|s|^{2^{*}-1}\right), \quad s \in \mathbb{R}, \quad x \in \mathbb{R}^{N} .
$$

We consider the mountain pass problem

$$
c(G ; \Omega) \stackrel{\text { def }}{=} \inf _{\varphi \in \Phi_{G, \Omega}} \sup _{t \geq 0} G\left(\varphi_{t}\right),
$$

where $\Phi_{G, \Omega} \subset \Phi_{G, \Omega}$ consists of paths with values in $\mathcal{D}_{0}^{1,2}(\Omega)$.

Remark 4.1. Regarding $\mathcal{D}_{0}^{1,2}(\Omega)$ as a closed subspace of $\mathcal{D}^{1,2}\left(\mathbb{R}^{N}\right)$, one can apply Theorem 6.1 to bounded sequences $u_{k} \in \mathcal{D}_{0}^{1,2}(\Omega) \subset \mathcal{D}^{1,2}\left(\mathbb{R}^{N}\right)$. The weak limits $w^{(n)}$ are of course not necessarily supported in $\Omega$. If, moreover, $|\Omega|<\infty$, then

(a) The set $\mathbb{N}_{0}$ consists only of the index 1 , since $u_{k}\left(\cdot-y_{k}\right) \rightarrow 0$ whenever $\left|y_{k}\right| \rightarrow \infty$.

(b) The set $\mathbb{N}_{-\infty}$ is empty since otherwise it easily follows that $\left\|u_{k}\right\|_{L^{2}} \rightarrow \infty$.

Theorem 4.2. Let $|\Omega|<\infty$. Assume in addition to (4.1) the condition $(\mu)$ (restricted to $x \in \Omega$ ). If

$$
c(G ; \Omega)<c\left(G_{+}\right),
$$

then the functional (3.2) has a critical point at the level $c(G ; \Omega)$ and every critical sequence at this level has a convergent subsequence.

Proof. The argument is completely analogous to the proof of Theorem 3.2, with the critical sequence regarded as a sequence in $\mathcal{D}^{1,2}\left(\mathbb{R}^{N}\right)$, and with obvious simplifications due to Remark 4.1 which leaves + as the only value for \#. 
Remark 4.3. Repetition of the proof of Theorem 3.2 also gives that if $F$ is like in Theorem 3.2, $\Omega \subset \mathbb{R}^{N}$ is a domain and inequalities (3.4) are satisfied, then the functional $G$ understood in restriction to $\mathcal{D}_{0}^{1,2}(\Omega)$ satisfies $(P S)_{c}$-condition at the mountain pass level $c(G ; \Omega)$ and thus has a critical point at this level.

Remark 4.4. Theorem 4.2 and Remark 4.3, unlike their counterpart on $\mathbb{R}^{N}$, cannot claim that $c(G ; \Omega)<c\left(G_{\#}\right)$ follows from $F(x, s)>F_{\#}(s)$, since the problem at infinity is supported on a different domain. Furthermore, [8] offers a counterexample for $N=3$ and $F(s)=|s|^{2^{*}}+\lambda u^{2}$ with sufficiently small positive $\lambda$.

On the other hand, if $\Omega$ is a bounded domain, $N>3$ and $F(x, s) \geq F_{+}(s)+\epsilon s^{2}$ with some $\epsilon>0$, then $c(G ; \Omega)<c\left(G_{+}\right)$. This follows by repetition of the estimates of Brezis and Nirenberg from [8] that involves the estimates for the minimizer from [18] by the Talenti minimizer.

Proposition 4.5. Under conditions of Theorem 4.2, $c(G ; \Omega) \leq c\left(G_{+}\right)$.

Proof. Let $w_{k} \in C_{0}^{\infty}(\Omega)$ be a sequence convergent in $\mathcal{D}^{1,2}\left(\mathbb{R}^{N}\right)$ to a critical point $w$ for $G_{+}$satisfying $G_{+}(w)=c\left(G_{+}\right)$.

$$
w_{s ; j, k}(x)=\gamma^{\frac{N-2}{2} j} w_{k}\left(\gamma^{j} x / s\right), \quad j \in \mathbb{N} .
$$

Assume without loss of generality that $0 \in \Omega$. Than for any $\epsilon>0, s \geq \epsilon$, and all $j$ sufficiently large, $w_{s ; j, k} \in C_{0}^{\infty}(\Omega)$. For $s<\epsilon$ we can redefine $w_{s ; j, k}$ as $\frac{s}{\epsilon} w_{\epsilon ; j, k}$. Then, with

$$
F_{t}(x, u)=t^{N} F\left(t x, t^{-\frac{N-2}{2}} u\right) \rightarrow F_{+}(u) \quad \text { as } \quad t \rightarrow 0 .
$$

and assuming, for each $k, \epsilon=\epsilon(k)$ sufficiently small, we have, similarly to the argument of Proposition 3.1,

$$
c(G) \leq \max _{s \geq 0} G\left(w_{s ; j, k}\right) \rightarrow \max _{s \geq 0} \frac{1}{2} s^{N-2}\left\|w_{k}\right\|^{2}-s^{N} \psi_{+}\left(w_{k}\right) .
$$

It is easy to see that as $k \rightarrow \infty$, the right hand side converges to

$$
\max _{s \geq 0} \frac{1}{2} s^{N-2}\|w\|^{2}-s^{N} \psi_{+}(w)=c\left(G_{+}\right) .
$$

It is also easy to see that $c(G ; \Omega) \leq c\left(G_{\#}\right)$ for general $\Omega$.

\section{Subcritical case}

Consider now the "positive mass" case $\lambda>0$ with the functional $\psi$ defined by expression (2.2) on the Sobolev space $H^{1}\left(\mathbb{R}^{N}\right), N \geq 1$, equipped with the equivalent norm

$$
\|u\|^{2}=\int_{\mathbb{R}^{N}}\left(\nabla|u|+\lambda|u|^{2}\right) d x .
$$

Let

$$
G(u)=\frac{1}{2}\|u\|_{H^{1}\left(\mathbb{R}^{N}\right)}^{2}-\psi(u) .
$$


We assume for every $\epsilon>0$ there exist $p_{\epsilon} \in\left(2,2^{*}\right)$ and $C_{\epsilon}>0$ such that

$$
|f(x, s)| \leq \epsilon\left(|s|+|s|^{2^{*}-1}\right)+C_{\epsilon}|s|^{p_{\epsilon}-1}, \quad s \in \mathbb{R}, \quad x \in \mathbb{R}^{N},
$$

or, for $N=1,2$,

$$
|f(x, s)| \leq \epsilon|s|+C_{\epsilon}|s|^{p_{\epsilon}-1}, \quad s \in \mathbb{R}, \quad x \in \mathbb{R}^{N},
$$

which assures that $G \in C^{1}\left(H^{1}\left(\mathbb{R}^{N}\right)\right)$. Assuming that the following uniform limit exists,

$$
f_{\infty}(s) \stackrel{\text { def }}{=} \lim _{|x| \rightarrow \infty} f(x, s),
$$

we define by analogy $F_{\infty}, \psi_{\infty}, G_{\infty}$, etc.

Proposition 5.1. Assume that (5.2) holds. Then

$$
c(G) \leq c\left(G_{\infty}\right) .
$$

Proof. Let $u_{t} \in \Phi_{G_{+}}$and let $y_{k} \in \mathbb{R}^{N},\left|y_{k}\right| \rightarrow \infty$. Then

$$
c(G) \leq \max _{t \geq 0} G\left(u_{t}\left(\cdot-y_{k}\right)\right) \rightarrow \max _{t \geq 0} G_{\infty}\left(u_{t}\right) .
$$

Minimizing the inequalities over all paths in $\Phi_{G_{+}}$we arrive at $c(G) \leq c\left(G_{\infty}\right)$.

Theorem 5.2. Assume (5.2) and $(\mu)$. If $c(G)<c\left(G_{\infty}\right)$, then every sequence $u_{k} \in$ $H^{1}\left(\mathbb{R}^{N}\right)$, such that $G^{\prime}\left(u_{k}\right) \rightarrow 0$ and $G\left(u_{k}\right) \rightarrow c(G)$, has a subsequence convergent to a critical point of $G$. Furthermore, the relation $c<c_{\infty}$ holds if

$$
\begin{aligned}
F(x, s) & \geq F_{\infty}(s), \\
x & \in \mathbb{R}^{N}, \quad \text { with the strong inequality in a neighborhood of } s=0 .
\end{aligned}
$$

Proof. The beginning of the proof is completely analogous to that for Theorem 3.2 and can be abbreviated. We apply Theorem 6.1 to the bounded critical sequence, noting, similarly to Remark 4.1 , that $\mathbb{N}_{-\infty}=\emptyset$ and, moreover, $\mathbb{N}_{+\infty}=\emptyset$ since $F_{+}=0$. With (6.6) taking the role of $(6.5)$ we arrive at an immediate analog of $(3.6)$ :

$$
G\left(w^{(1)}\right)+\sum_{n \geq 2} G_{\infty}\left(w^{(n)}\right) \leq c(G)<c\left(G_{\infty}\right) .
$$

As in the proof of Theorem 3.2, all the terms in the left hand side are non-negative due to $(\mu)$. Assume that there exists $m \geq 2$ such that $w^{(m)} \neq 0$. Observe the path $t \mapsto w^{(m)}(\cdot / t)$ is of the class $\Phi_{G_{\infty}}$. Indeed, its continuity in $\mathcal{D}^{1,2}\left(\mathbb{R}^{N}\right)$ norm was shown in Proposition 2.1, and the proof of remaining continuity, in $L^{2}$ norm is analogous. The nonlinearity satisfies the requirements of [5] for Pohožaev identity (1.5). Thus, since

$$
\int_{\mathbb{R}^{N}}\left(F_{\infty}\left(w^{(m)}\right)-\lambda\left|w^{(m)}\right|^{2}\right) d x=\frac{1}{2^{*}}\left\|w^{(m)}\right\|_{\mathcal{D}^{1,2}\left(\mathbb{R}^{N}\right)}^{2}>0
$$

the functional $G_{\infty}$ on the path $t \mapsto w^{(m)}(\cdot / t)$,

$$
G_{\infty}\left(w^{(m)}(\cdot / t)\right)=\frac{1}{2} t^{N-2}\left\|w^{(m)}\right\|_{\mathcal{D}^{1,2}\left(\mathbb{R}^{N}\right)}^{2}-t^{N} \int_{\mathbb{R}^{N}}\left(F_{\infty}\left(w^{(m)}\right)-\lambda\left|w^{(m)}\right|^{2}\right) d x,
$$


converges to $-\infty$ when $t \rightarrow \infty$. Furthermore, the maximum of this expression over $t \geq 0$ is clearly attained at a single point, which is necessarily $t=1$, since $\left(G_{\infty}^{\prime}\left(w^{(m)}\right), w^{(m)}\right)=0$. Thus

$$
c\left(G_{\infty}\right) \leq \max _{t \geq 0} G_{\infty}\left(w^{(m)}(\cdot / t)\right)=G_{\infty}\left(w^{(m)}\right) d x .
$$

On the other hand, since, $G_{\infty}\left(w^{(n)}\right) \geq 0$ for all $n>1$ and $G\left(w^{(1)}\right) \geq 0$, we have from $(5.5)$

$$
G_{\infty}\left(w^{(m)}\right) \leq c(G)<c\left(G_{\infty}\right)
$$

arriving to a contradiction with (5.6) unless $w^{(n)}=0$ for all $n \geq 2$. This, via the usual convergence argument, yields $u_{k} \rightarrow w^{(1)}$. The implication $(5.4) \Rightarrow c(G)<$ $c\left(G_{\infty}\right)$ is immediate once we take into account that the range of any function in $H_{0}^{1}(\Omega)$ is connected and contains zero.

Remark 5.3. Theorem 5.2 can be trivially generalized to the case of periodic coefficients. Let $V \in L^{\infty}\left(\mathbb{R}^{N}\right), V>0$, be $\mathbb{Z}^{N}$-periodic, that is satisfy

$$
V(x+y)=V(x) \quad \text { for all } \quad x \in \mathbb{R}^{N}, \quad y \in \mathbb{Z}^{N} .
$$

We may equip $H^{1}\left(\mathbb{R}^{N}\right)$ with an equivalent norm

$$
\|u\|^{2}=\int_{\mathbb{R}^{N}}\left(|\nabla u|^{2}+V(x) u^{2}\right) d x .
$$

Assume that there is a function $f_{\infty} \in C\left(\mathbb{R}^{N} \times \mathbb{R}\right), \mathbb{Z}^{N}$-periodic in the first argument, such that $f\left(x+y_{k}, s\right) \rightarrow f_{\infty}(x, s)$ for any sequence $y_{k} \in \mathbb{Z}^{N},\left|y_{k}\right| \rightarrow \infty$. Theorem 5.2 remains true also under these modifications.

\section{Appendix: Weak convergence decomposition in $\mathcal{D}^{1,2}\left(\mathbb{R}^{N}\right)$}

The following theorem is Theorem 5.1 from [34], with the dilation factor 2 replaced by general $\gamma$.

Theorem 6.1. Let $u_{k} \in \mathcal{D}^{1,2}\left(\mathbb{R}^{N}\right), N>2$, be a bounded sequence. Let $\gamma>1$. There exist $w^{(n)} \in \mathcal{D}^{1,2}\left(\mathbb{R}^{N}\right), y_{k}^{(n)} \in \mathbb{R}^{N}, j_{k}^{(n)} \in \mathbb{Z}$ with $k, n \in \mathbb{N}$, and disjoint sets $\mathbb{N}_{0}, \mathbb{N}_{+\infty}, \mathbb{N}_{-\infty} \subset \mathbb{N}$, such that, for a renumbered subsequence of $u_{k}$,

$$
\begin{aligned}
& w^{(n)}=w \text {-lim } \gamma^{-\frac{N-2}{2} j_{k}^{(n)}} u_{k}\left(\gamma^{-j_{k}^{(n)}} \cdot+y_{k}^{(n)}\right), \quad n \in \mathbb{N}, \\
& \left|j_{k}^{(n)}-j_{k}^{(m)}\right|+\left|\gamma_{k}^{j_{k}^{(n)}}\left(y_{k}^{(n)}-y_{k}^{(m)}\right)\right| \rightarrow \infty \quad \text { for } \quad n \neq m, \\
& \sum_{n \in \mathbb{N}}\left\|w^{(n)}\right\|_{\mathcal{D}^{1,2}}^{2} \leq \limsup \left\|u_{k}\right\|_{\mathcal{D}^{1,2}}^{2}, \\
& u_{k}-\sum_{n \in \mathbb{N}} \gamma^{\frac{N-2}{2} j_{k}^{(n)}} w^{(n)}\left(\gamma^{j_{k}^{(n)}}\left(\cdot-y_{k}^{(n)}\right)\right) \rightarrow 0, \quad \text { in } \quad L^{2^{*}}\left(\mathbb{R}^{N}\right),
\end{aligned}
$$


and the series above converges uniformly in $k$. Furthermore, $1 \in \mathbb{N}_{0}, y_{k}^{(1)}=0$; $j_{k}^{(n)}=0$ whenever $n \in \mathbb{N}_{0} ; j_{k}^{(n)} \rightarrow-\infty$ (resp. $\left.j_{k}^{(n)} \rightarrow+\infty\right)$ whenever $n \in \mathbb{N}_{-\infty}$ (resp. $n \in \mathbb{N}_{+\infty}$ ); and $y_{k}^{(n)}=0$ whenever $\left|\gamma^{j_{k}^{(n)}} y_{k}^{(n)}\right|$ is bounded.

The following statements are, respectively, Lemma 5.6 (an elementary modification) and Remark 3.4 from [34].

Lemma 6.2. Let $F \in C\left(R^{N} \times \mathbb{R}\right)$ satisfy $\mid F\left(x,\left.s|\leq c| s\right|^{2^{*}}\right.$, let $\gamma>0, N>2$, and assume that the following limits exist and are uniform in $x \in \mathbb{R}^{N}$ :

$$
\begin{aligned}
& F_{+}(s) \stackrel{\text { def }}{=} \lim _{j \in \mathbb{Z}, j \rightarrow+\infty} \gamma^{-N J} F\left(\gamma^{-j} x, \gamma^{\frac{N-2}{2}} j\right), \\
& F_{-}(s) \stackrel{\text { def }}{=} \lim _{j \in \mathbb{Z}, j \rightarrow-\infty} \gamma^{-N J} F\left(\gamma^{-j} x, \gamma^{\frac{N-2}{2}} j\right), \\
& F_{0}(s) \stackrel{\text { def }}{=} \lim _{|x| \rightarrow \infty} F(x, s) .
\end{aligned}
$$

Let $u_{k} \in \mathcal{D}^{1,2}\left(\mathbb{R}^{N}\right), w^{(n)}, y_{k}^{(n)} \in \mathbb{R}^{N}$ and let $j_{k}^{(n)} \in \mathbb{Z}, \mathbb{N}_{0}, \mathbb{N}_{+\infty}, \mathbb{N}_{-\infty} \subset \mathbb{N}$ be as provided by Theorem 6.1. Then

$$
\begin{aligned}
& \lim _{k \rightarrow \infty} \int_{\mathbb{R}^{N}} F\left(u_{k}\right)= \\
& \quad \sum_{n \in \mathbb{N}_{0}} \int_{\mathbb{R}^{N}} F_{0}\left(w^{(n)}\right)+\sum_{n \in \mathbb{N}_{+\infty}} \int_{\mathbb{R}^{N}} F_{+}\left(w^{(n)}\right)+\sum_{n \in \mathbb{N}_{-\infty}} \int_{\mathbb{R}^{N}} F_{-}\left(w^{(n)}\right) .
\end{aligned}
$$

Lemma 6.3. Let $F \in C\left(R^{N} \times \mathbb{R}\right)$ satisfy 5.2, 5.3, and assume that the following uniform limit exists:

$$
F_{\infty}(s) \stackrel{\text { def }}{=} \lim _{|x| \rightarrow \infty} F(x, s) .
$$

Let $u_{k} \in H^{1}\left(\mathbb{R}^{N}\right), w^{(n)}, y_{k}^{(n)} \in \mathbb{R}^{N}$ be as provided by Theorem 6.1 with $\mathbb{N}_{ \pm}=\emptyset$. Then

$$
\lim _{k \rightarrow \infty} \int_{\mathbb{R}^{N}} F\left(u_{k}\right)=\sum_{n \in \mathbb{N}} \int_{\mathbb{R}^{N}} F_{\infty}\left(w^{(n)}\right) .
$$

\section{Acknowledgements}

The author thanks Moshe Marcus and Ian Schindler for their encouraging remarks. He is grateful for the warm hospitality extended to him by B. Alziary, J. Fleckinger and I. Schindler at Ceremath - Toulouse 1.

\section{References}

[1] A. Ambrosetti, P. H. Rabinowitz, Dual variational methods in critical point theory and applications. J. Functional Analysis 14 (1973), 349-381. 
[2] T. Bartsch, Z.-Q. Wang, M. Willem, The Dirichlet problem for superlinear elliptic equations. Stationary partial differential equations vol. 2, 1-55 (2005), Handb. Differ. Equ., Elsevier, North Holland, Amsterdam.

[3] T. Bartsch, Z. Q. Wang, Existence and multiplicity results for some superlinear elliptic problems on $R^{N}$. Comm. Partial Differential Equations 20 (1995), 1725-1741.

[4] V. Benci, G. Cerami, Existence of positive solutions of the equation $-\Delta u+a(x) u=$ $u^{(N+2) /(N-2)}$ in $\mathbb{R}^{N}$, J. Func. Anal. 88 (1990) 90-117.

[5] H. Berestycki, P.-L. Lions, Nonlinear scalar fields equations I, Existence of a ground state Arch. Rat. Mech. Anal. 82 (1983), 313-346.

[6] G. Bliss, An integral inequality, J. London Math. Soc. 5 (1930), 44-46.

[7] H. Brézis, J. M. Coron, Convergence of solutions of H-systems or how to blow bubbles, Archive Rat. Mech. Anal. 89 (1985), 21-56.

[8] H. Brézis, L. Nirenberg, Positive solutions of an elliptic equation with a nonlinearity involving critical Sobolev exponent, Comm. Pure Appl. Math. 36 (1983), 437-476.

[9] H. Brezis, E. Lieb, A relation between pointwise convergence of functions and convergence of functionals, Proc. Amer. Math. Soc. 88 (1983), 486-490.

[10] J. Chabrowski, Concentration-compactness principle at infinity and semilinear elliptic equations involving critical and subcritical Sobolev exponents, Calc. Var. 3 (1995), 493-512.

[11] G. Cerami, D. Fortunato, M. Struwe, Bifurcation and multiplicity results for nonlinear elliptic problems involving critical Sobolev exponents, Ann. Inst. H. Poincaré Anal. Non Linéaire 1 (1984), 341-350.

[12] J. Chabrowski, A. Szulkin, On a semilinear Schrödinger equation with critical Sobolev exponent. Proc. Amer. Math. Soc. 130 (2002), no. 1, 85-93 (electronic).

[13] J. Chabrowski, Weak convergence methods for semilinear elliptic equations. World Scientific Publishing Co., Inc., River Edge, NJ, 1999.

[14] J. Chabrowski, J. Yang, Existence theorems for elliptic equations involving supercritical Sobolev exponent Advances in Differential Equations, 2 (1997), 231-256.

[15] V. Coti Zelati, Critical point theory and applications to elliptic equations in $R^{n}$. (English summary) Nonlinear functional analysis and applications to differential equations (Trieste, 1997), 102-121, World Sci. Publ., River Edge, NJ, 1998.

[16] M. Del Pino, P. Felmer, Least energy solutions for elliptic equations in unbounded domains, Proc. Royal Soc. Edinburgh 126A (1996), 195-208.

[17] M. J. Esteban, P.-L. Lions, A compactness lemma, Nonlinear Anal. 7 (1983), 381385 .

[18] M. Flucher, S. Müller, Radial symmetry and decay rate of variational ground states in the zero mass case, SIAM J. Math. Anal. 20, 712-719 (1998)

[19] M. Flucher, S. Müller, Concentration of low energy extremals, Ann. Inst. H. Poincaré - Analyse non-lineaire 16, 269-298 (1999).

[20] M. Flucher, Variational problems with concentration, Progress in Nonlinear Differential Equations and their applications36, Birkhäuser 1999.

[21] E. Lieb, On the lowest eigenvalue of the Laplacian for the intersection of two domains. Invent. Math. 74 (1983), 441-448. 
[22] P.-L. Lions, The concentration-compactness principle in the calculus of variations. The locally compact case, part 1 . Ann. Inst. H. Poincare, Analyse non linéaire 1 (1984), 109-1453.

[23] P.-L. Lions, The concentration-compactness principle in the calculus of variations. The locally compact case, part 2. Ann. Inst. H. Poincare, Analyse non linéaire 1 (1984), 223-283.

[24] P.-L. Lions, The concentration-compactness principle in the calculus of variations. The limit case, part 2, Revista Matematica Iberoamericana, 1.1 (1985), 145-201.

[25] P.-L. Lions, The concentration-compactness principle in the calculus of variations. The limit case, part 2, Revista Matematica Iberoamericana 1.2 (1985), 45-121.

[26] P.-L. Lions, Solutions of Hartree-Fock equations for Coulomb systems, Comm. Math. Phys. 109 (1987), 33-97.

[27] S. I. Pohožaev, Eigenfunctions of the equation $\Delta u+\lambda f(u)=0$. Sov. Math. Doklady 5 (1965), 1408-1411.

[28] P. H. Rabinowitz, On a class of nonlinear Schrödinger equations. Z. Angew. Math. Phys. 43 (1992), 270-291.

[29] I. Schindler, K. Tintarev, An abstract version of the concentration compactness principle, Revista Mat. Complutense 15 (2002), 1-20.

[30] B. Sirakov, Existence and multiplicity of solutions of semi-linear elliptic equations in $\mathbb{R}^{N}$. Calc. Var. Partial Differential Equations 11 (2000), 119-142.

[31] M. Struwe, A global compactness result for elliptic boundary value problems involving limiting nonlinearities, Math. Z. 187 (1984), 511-517.

[32] G. Talenti, Best constant in Sobolev inequality, Ann. Mat. Pura Appl. (4) 110 (1976), 353-372.

[33] K. Tintarev, Concentration-compactness principle for mountain pass problems, in Proceedings of Conference on Differential and Difference Equations, August 1-5, 2005, Melbourne, Florida, Hindawi Publishing Corporation, 2006.

[34] K. Tintarev, K.-H. Fieseler, Concentration compactness: functional-analytic grounds and applications, Imperial College Press 2007.

[35] M. Willem, Minimax theorems. Progress in Nonlinear differential equations and their applications, 24. Birkhäuser Boston, Inc., Boston, MA, 1996.

Kyril Tintarev

Department of Mathematics

Uppsala University

P.O.Box 480

S-751 06 Uppsala

Sweden

e-mail: kyril.tintarev@math.uu.se

Received: 23 September 2007.

Accepted: 25 April 2008. 